MATEC Web of Conferences 22,03006 (2015)

DOI: $10.1051 /$ matec conf/ 20152203006

(C) Owned by the authors, published by EDP Sciences, 2015

\title{
Parameterized Analysis of 2-DOF Motion Platform Based on ADAMS
}

\author{
Hanyuan $\mathrm{Hu}$ \\ Department of Mechanical Engineering Changchun, Changchun University of Science and Technology, \\ Changchun, Jilin, China \\ Lizhong Zhang * \\ Fundamental Science on Space-ground Laser Communication Technology Laboratory, Changchun, Jilin, China
}

Yangyang Bai \& Jin Hong

Department of Mechanical Engineering Changchun, Changchun University of Science and Technology, Changchun, Jilin, China

\begin{abstract}
According to the functions of parametric modeling and analysis from ADAMS, this thesis was established a parametric simulation model in order to optimize the rated output power of electric cylinders according to the real field environment. First, the variable which could affect sensitivity of the output variables was chosen by the electric cylinder's elongation which was obtained through loop vector method. Then this thesis tried to get the optimum optimization design parameters through the simulation, and the change of rated output power affected by the change of parameters, meanwhile, made a filter and calibration of parameters which have greater influence on sensibilities. The goal of design could meet the qualification with less work load and faster speed. It is concluded that the change of the location parameters affects the rated output power.
\end{abstract}

Keyword: 2-DOF motion platform; ADAMS; parameterized analysis; simulation

\section{INTRODUCTION}

Nowadays, the parametric design was an emphasis in the study of $\mathrm{CAD}^{[1]}$. The MSC ADAMS possesses the functions of parametric modeling and analyzing in order to improve the efficiency of mechanism design and shorten the designing period. The document ${ }^{[2]}$ regarded the minimum driving angles as an optimization goal, and the lengths of links are parameterized via ADAMS by creating design parameters. The document ${ }^{[3]}$ regarded the minimum base driving torque as an optimization goal to get the optimal solution of substructure driving torque. The document ${ }^{[4]}$ chose side way displacement as an optimization goal to finish parametric optimization.

The motion platform was used to be tested in field environment. It required that the rated output power of the electric cylinder should be as low as better, so it could meet the movement's demand which was due to power constrains in the field. So that it could reduce the requirement of power supply. Also, we should research the structure of motion platform deeper because every parameters of platform have different effects on the electric cylinder power. The location parameter as a very important design factor in the design process determined the motion platform's characteristics. So it was necessary to study on the relationship between motion platform and characteristics. The traditional method of analysis to the model which had modified until meeting the desired model *Corresponding author: zlzcust@ 126. .com and results was a mindless and repetitive task. And the method used in this paper was the first parametric model which was established by the modeling, analysis simulation and post processing module; and the rated output power of electric cylinder was lower by the above analysis and on the premise of meeting the practice; then we obtained the expected result which could reduce the requirement of power.

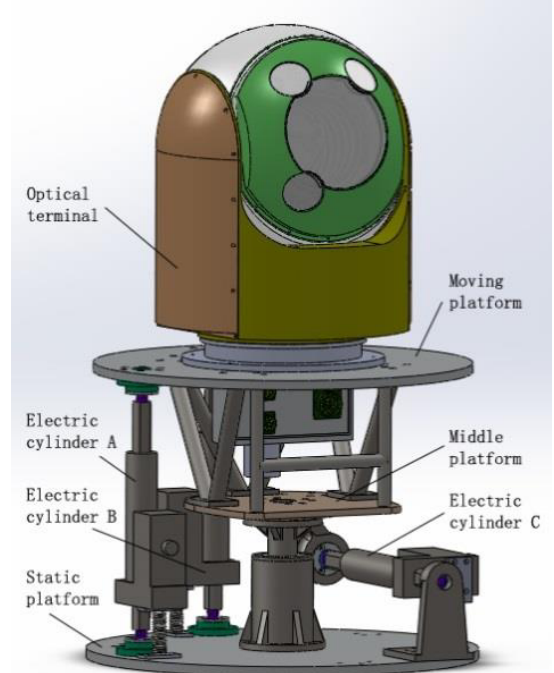

Figure 1. Structure of 2-DOF motion platform

This is an Open Access article distributed under the terms of the Creative Commons Attribution License 4.0, which permits unrestricted use, distribution, and reproduction in any medium, provided the original work is properly cited. 


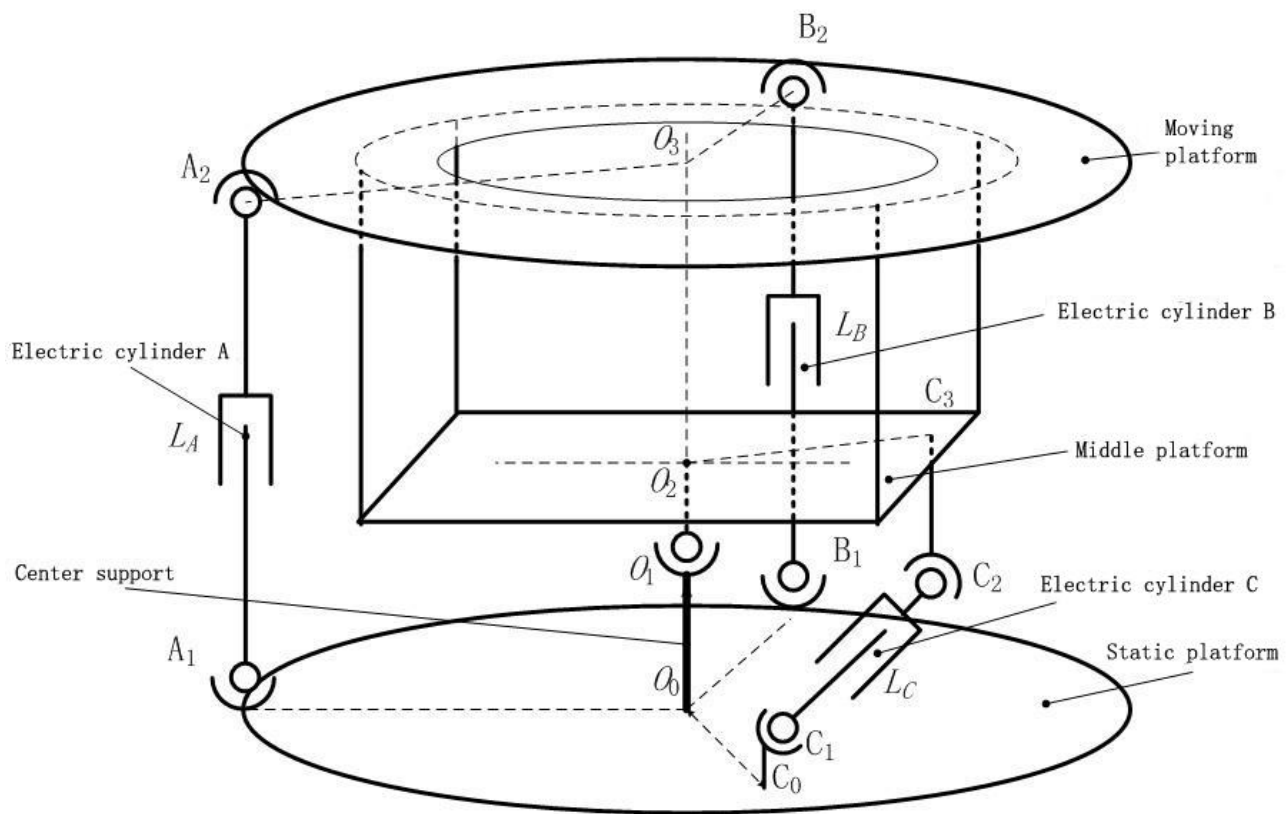

Figure 2. Schematic diagram of 2-DOF motion platform

\section{INTRODUCTION OF 2-DOF MOTION PLAT- FORM'S STRUCTURE}

As shown in Figure 1, the 2-DOF motion platform consists of moving platform, middle supporting platform, static platform, servo electric cylinder, hinges and so on. The servo electric cylinder as A,B shows parallel with the central axis $\mathrm{O} 0 \mathrm{O} 1 \mathrm{O} 2 \mathrm{O} 3$ of platform and it was vertical to servo electric cylinder $C$; the moving platform was connected with static platform by middle platform; cylinders were connected with moving and static platform by hinges; platform moves through the servo electric cylinders.

Stabilized the optical terminal on the moving platform; it simulated attitude change on board by controlling three electric cylinders' telescopic motion of the azimuth and elevation. The moving platform and middle supporting platform were connected by structure of frames. This scheme not only increases space to install equipment, but decreases the height of equipment's center of gravity to improve the equipment stability as well. It cuts the size of the motion platform then lightens the weight of the whole platform on the premise which meets the demand of enough stiffness. Because of the motion platform moved by the way supported by middle and moved by two electric cylinders and maintenance-free bearing in the middle support nearly most loads, therefore, it reduces the demand for electric cylinder's output torque. Furthermore, it meets the low power requirements which chosen in field environment test.

\section{PARAMETERIZED MODELING OF MOTION PLARFORM}

\subsection{Selection of the design variables}

Simplify the motion platform for the convenience of parameterized modeling as shown in Figure 2, the selection of the design variables affected the performance of the prototype ${ }^{[5-6]}$, which was the main content for parametric design, and it had great influence on the motion platform. The variable which could affect sensitivity of the output variables was chosen by the electric cylinder's elongation which was got through the loop vector method.

Based on the analysis of motion platform, the model of electric cylinder was parted from the motion platform as shown in Figure 3.

Coordinate system $\mathrm{O}_{0}-\mathrm{X}_{0} \mathrm{Y}_{0} \mathrm{Z}_{0}$ was established. Three key points in the coordinate system were $\mathrm{O}_{0}(0$, $0,0), B_{1}\left(R_{0}, 0,0\right), O_{1}(0,0, q) . R_{0}$ was the radius of flexural pivot on static platform and $q$ was vertical distance between the rotation center of middle support's hinge and static platform. R0 and q are constant.

The point $\mathrm{B}_{2}$ 's coordinate was $\left(\mathrm{r}_{0} \cos \lambda_{2}, 0, \mathrm{r}_{0} \sin \lambda_{2}\right)$ in moving coordinate system $\mathrm{O}_{1}-\mathrm{X}_{1} \mathrm{Y}_{1} \mathrm{Z}_{1}$ based on an initial state. $\mathrm{r} 0$ is a linear distance between flexural pivot $\mathrm{B}_{2}$ and hinge $\mathrm{O}_{1} . \lambda_{2}$ was angle between $\overrightarrow{O_{1} B_{2}}$ and $+\mathrm{X}_{1}$.

According to the principle of coordinate changed, the position vector $P^{\prime}$ in moving coordinate system could be transformed to position vector $P$ in static coordinate system through the transform formula, which was shown as follows:

$\mathrm{P}=\mathrm{T} \cdot \mathrm{P}^{\prime}+\mathrm{P}_{0}$ 


\section{ICETA 2015}

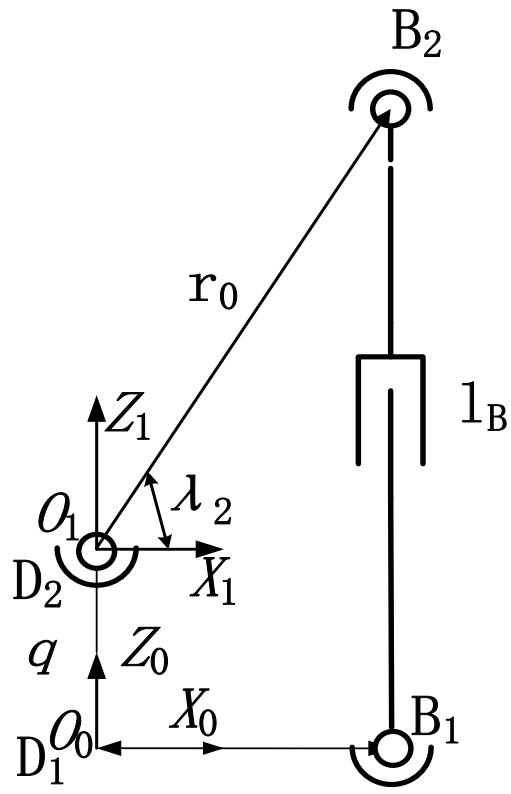

Figure 3. Model of electric cylinder

Besides, $\mathrm{T}$ was the direction cosine matrix, $\mathrm{P}_{0}$ was the position vector of a moving coordinate system in static coordinate system.

Besides, $\quad P_{B_{2}}^{\prime}=\left[\begin{array}{llll}r_{0} \cos \lambda_{2} & 0 & r_{0} \sin \lambda_{2}\end{array}\right]^{T}$ $P_{B_{2}}^{\prime}=\left[\begin{array}{llll}r_{0} \cos \lambda_{2} & 0 & r_{0} \sin \lambda_{2}\end{array}\right]^{T}$. Insert them into formula mentioned earlier.

$p_{B_{2}}=\left(t_{11} r_{0} \cos \lambda_{2}+t_{13} r_{0} \sin \lambda_{2}, t_{21} r_{0} \cos \lambda_{2}\right.$

$\left.+t_{23} r_{0} \sin \lambda_{2}, t_{31} r_{0} \cos \lambda_{2}+t_{33} r_{0} \sin \lambda_{2}+q\right)$

Therefore, the elongation of electric cylinder B is shown as follows:

$L_{B}=\sqrt{\Delta X_{B}^{2}+\Delta Y_{B}^{2}+\Delta Z_{B}^{2}}$

Insert the coordinate of $\mathrm{B} 1$ into $\mathrm{L}_{\mathrm{B}}$ :

$\Delta X_{B}^{2}=\left(\mathrm{t}_{11} r_{0} \cos \lambda_{2}+t_{13} r_{0} \sin \lambda_{2}-R_{0}\right)^{2} ;$

$\Delta Y_{B}^{2}=\left(\mathrm{t}_{21} r_{0} \cos \lambda_{2}+t_{23} r_{0} \sin \lambda_{2}\right)^{2}$

$\Delta Z_{B}^{2}=\left(\mathrm{t}_{31} r_{0} \cos \lambda_{2}+t_{33} r_{0} \sin \lambda_{2}+q\right)^{2} ;$

Relational expression of electric cylinder C's elongation is obtained as the same as that mentioned earlier. We had solved the expression which is up to the rod length in linear regression way. Furthermore, we evaluated the differential divisor variable between input and output by the LINGO software.

The variable which could affect sensitivity of the output variables must be chosen for parametric modeling of motion platform. So eight key points were chosen in this paper, which were the center point $\mathrm{O} 1$ of moving platform, the center point $\mathrm{O} 2$ of moving platform, and the end points $\mathrm{A}_{1}, \mathrm{~A}_{2}, \mathrm{~B}_{1}, \mathrm{~B}_{2}, \mathrm{C}_{1}$, and $\mathrm{C}_{2}$ on the three electric cylinders. This points were reference points, which corresponded to eight design variables, named DV_1 DV_8.

Table 1. Coordinates of reference points

\begin{tabular}{|l|l|l|l|}
\hline & Loc_X & Loc_Y & Loc_Z \\
\hline O1 & 0 & DV_3 & 0 \\
\hline O2 & 0 & DV_4 & 0 \\
\hline $\mathrm{A}_{1}$ & DV_1 & 0 & 0 \\
\hline $\mathrm{A}_{2}$ & DV_1 & DV_2 & 0 \\
\hline $\mathrm{B}_{1}$ & 0 & 0 & DV_1 \\
\hline $\mathrm{B}_{2}$ & 0 & DV_2 & DV_1 \\
\hline $\mathrm{C}_{1}$ & DV_5 & DV_6 & DV_7 \\
\hline $\mathrm{C}_{2}$ & DV_5 & DV_6 & DV_8 \\
\hline
\end{tabular}

3.2 Established the parametric model of motion platform

The simplified model was established by modeling tool in ADAMS, which could make parametric design for the motion platform. In the process of modeling, the point could be moved to change the layout of electric cylinder through location parameter of the motion platform by establishing the point. Figure 4 showed the model according to the point coordinates:

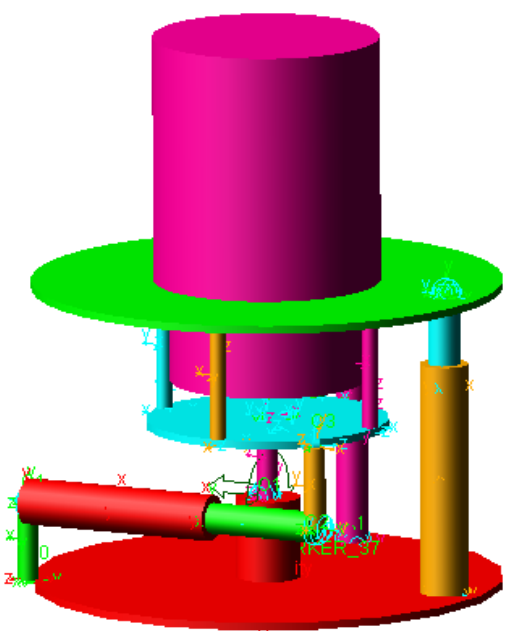

Figure 4. Model of 2-DOF motion platform 


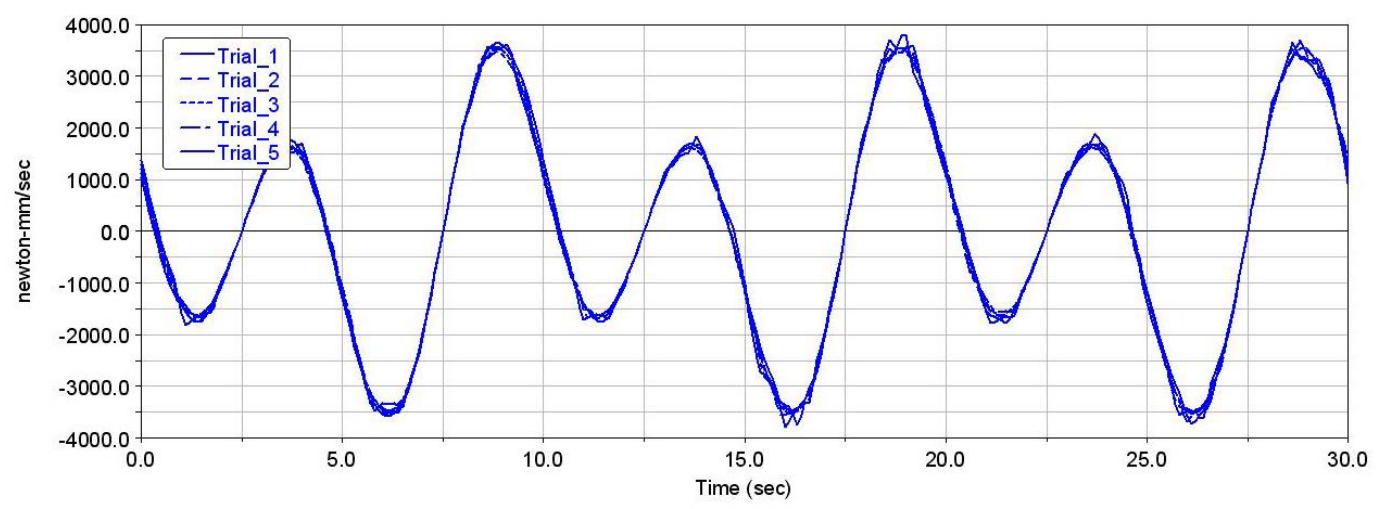

Figure 5. Influence law of variable DV_1 to the power of electric cylinder B

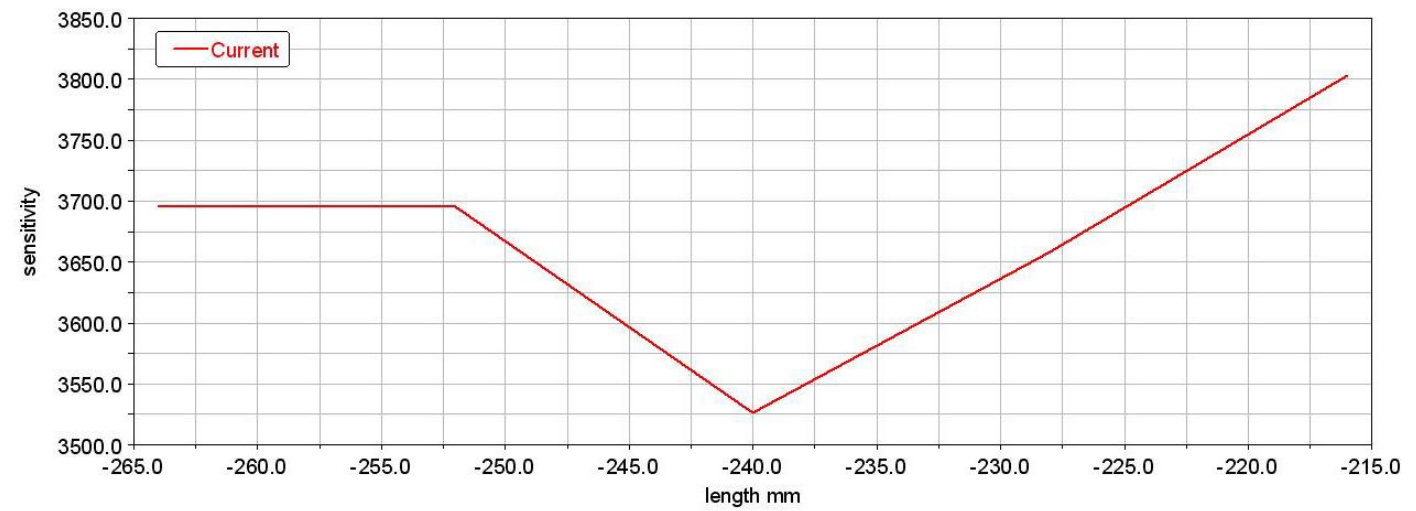

Figure 6. Sensitivity law of the variable DV_1 to the power of electric cylinder B

Designed variables DV 1 DV 8 in ADMAS which are the coordinate parameters of electric cylinder, and set the range of design variable which is $\pm 10 \%$.

Table 2. Coordinate of design variable's starting value

\begin{tabular}{|l|l|l|}
\hline Design variable & Starting value & Range \\
\hline DV_1 & -240.0 & $-10.0,10.0$ \\
\hline DV_2 & 389.2 & $-10.0,10.0$ \\
\hline DV_3 & 104.2 & $-10.0,10.0$ \\
\hline DV_4 & 207.7 & $-10.0,10.0$ \\
\hline DV_5 & 137.06 & $-10.0,10.0$ \\
\hline DV_6 & 101.7 & $-10.0,10.0$ \\
\hline DV_7 & 260.55 & $-10.0,10.0$ \\
\hline DV_8 & -129.45 & $-10.0,10.0$ \\
\hline
\end{tabular}

\section{RESEARCH ABOUT THE SENSITIVITY OF STRUCTURE PARAMETER}

4.1 Research about the sensitivity of structure parameter

The study about sensitivity of structure's parameters was mainly about the influenced degree of sensitivity of single design variable on the overall prototype. The design variables were analyzed in the set range, which had effects on sensibilities to output power. Making a filtering and revising of parameters which have greater influence on sensibilities could shorten the computation time and save the development cost.

It regarded the electric cylinder's power dissipation as an object function, meanwhile, it regarded structure's parameters as design variable, furthermore, it would evaluate by the index of sensitivity.

$S_{i}=\frac{1}{2}\left(\frac{O_{i+1}-O_{i}}{V_{i+1}-V_{i}}+\frac{O_{i}-O_{i-1}}{V_{i}-V_{i-1}}\right)$

In type: $O-$-desired value; 
ICETA 2015

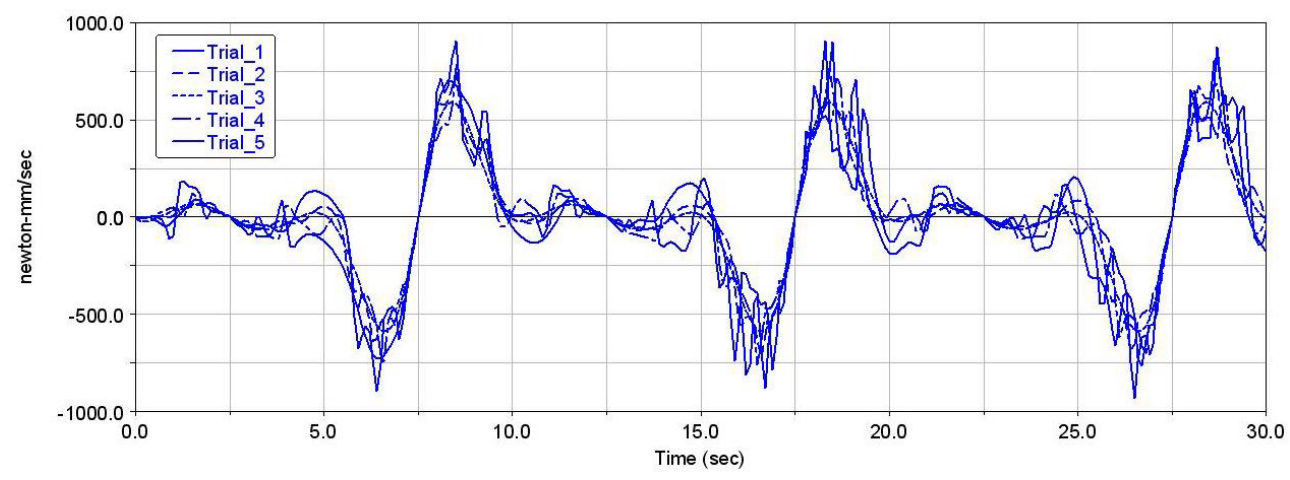

Figure 7. Influence law of variable DV_1 to the power of electric cylinder C

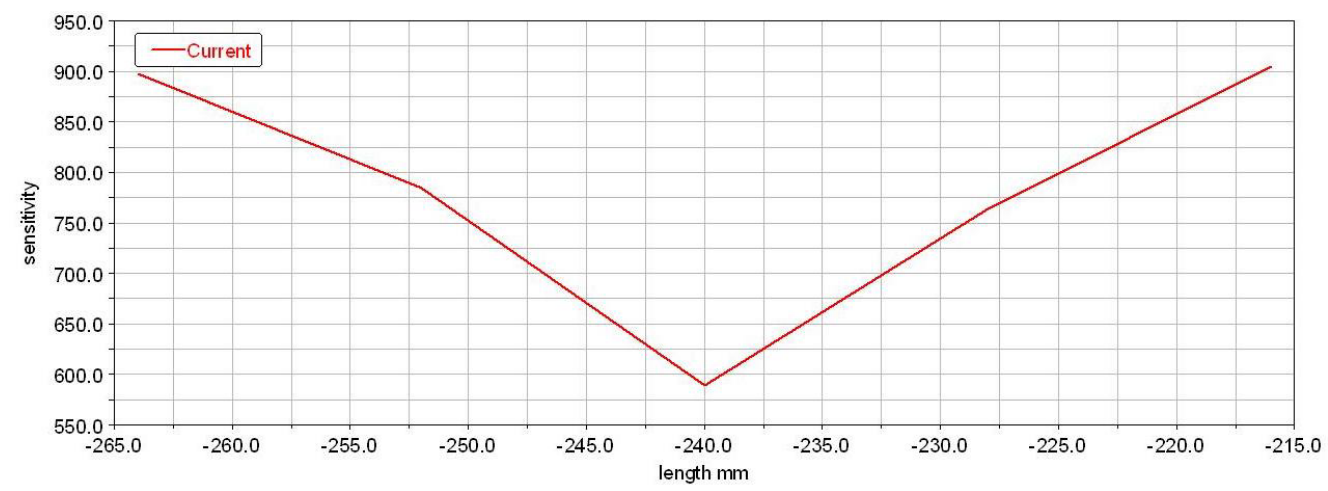

Figure 8 . Sensitivity law of the variable $\mathrm{DV}_{-} 1$ to the power of electric cylinder C

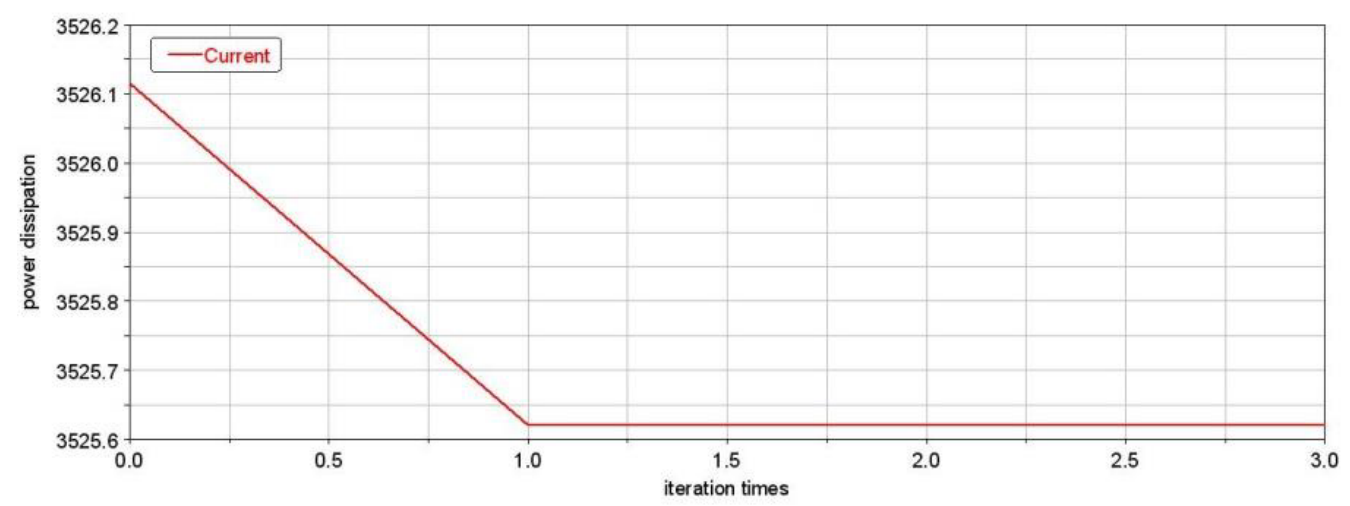

Figure 9. Platform's power optimization curve

$V$ - design parameter;

$i$ - - iteration times;

This paper mainly evaluated the electric cylinder B and $\mathrm{C}$ due to the servo electric cylinder $\mathrm{A}$ was a supplementary role in the movement and stressed less than the other two electric cylinders. It took $\mathrm{X}$-coordinate of hinge $\mathrm{A}_{1}$ variables $\mathrm{DV}_{-} 1$ as an exam- ple to introduce the calculation about sensitivity of the single design variable.

First of all, the number of iteration times was 5 . Then we set the maximum power consumption of electric cylinder as the target and design parameter values as DV 1:

Figure 5 to Figure 8 depicted that the research about 


\section{MATEC Web of Conferences}

the influence law of platform to power of electric cylinder B,C and law of each size's sensitivity as following variable DV_1's change. It could be used to calculate that the sensitivity of variable DV 1 to electric cylinder $\mathrm{C}$ was 0.83 , and that one to electric cylinder $\mathrm{C}$ was 1.53 in the initial position. The larger sensitivity is, the greater design variable influences of output power of the electric cylinder are. Therefore, the change of the electric cylinder B must be considered before changing the value of DV 1 . Similarly, sensitivity of the electric cylinder affected by 8 parameters was got through the simulation, and the results are shown in Table 3 :

Table 3. Sensitivity of electric cylinder $\mathrm{C}$ about design variable

\begin{tabular}{|l|l|l|l|}
\hline $\begin{array}{l}\text { Design } \\
\text { variable }\end{array}$ & Starting & $\begin{array}{l}\text { The sensitivity of } \\
\text { electric cylinder B }\end{array}$ & $\begin{array}{l}\text { The sensitivity of } \\
\text { electric cylinder C }\end{array}$ \\
\hline DV_1 & -240.0 & 1.53 & 0.83 \\
\hline DV_2 & 389.2 & 0.15 & 0.19 \\
\hline DV_3 & 104.2 & 0 & 0 \\
\hline DV_4 & 207.7 & 0.59 & 0.96 \\
\hline DV_5 & 137.06 & 8.14 & 5.62 \\
\hline DV_6 & 101.7 & 10.91 & 5.40 \\
\hline DV_7 & 260.55 & 1.61 & 0.14 \\
\hline DV_8 & -129.45 & 1.41 & 0.14 \\
\hline
\end{tabular}

According to the analysis of the key parameters in Table 3, the result could be obtained that DV 1, DV 5 and DV 6 had great influence on the simulation platform of the overall power consumption. Therefore, three design variables were chosen to analyze again as the final optimization variables could be used to keep the platform optimizing. The installation position of electric cylinder $\mathrm{C}$ had great influence on output power, so it was a main factor in the design. Others weren't treated as optimization design variables which sensitivity is smaller and had little effects on output power.

\subsection{Optimizing structure parameters of the platform}

Due to the structure parameters, the servo electric cylinder $\mathrm{C}$ was more sensitive than electric cylinder $\mathrm{B}$. Then the following was regarded the minimum power of electric cylinder B as the optimization objective function by iterative optimization design. According to the trend of variable sensitivity, the definition change interval was that DV 1 was $[-245,-235]$; DV 5 was $[130,142]$; DV 6 was $[95,105]$.

Figure 9 showed the iterative optimization process curve, and the optimization results were: DV $1=240.12$; DV 5=136.74; DV $6=100.46$; the power consumption was the lowest. Then we could determine the electric cylinder's position.

\section{CONCLUSION}

Through making uses of design optimization function from ADAMS/View, this paper regarded electric cylinder power as the objective function to optimize the design, optimize the evaluation results by evaluate the sensitive of structure parameters, and determine various electric cylinder position. At the same time, with analyzing of the model parameters, it further understood the influence of structure parameters on the performance of the prototype, and then the sensitive parameters were optimized. Through further analysis, this paper obtained the reasonable design parameters. On the premise of meeting the requirements, the rated output power of electric cylinder was lower by the above analysis. Then it obtained the expected result which could reduce the requirement of power.

\section{REFERENCES}

[1] Jin Jianguo, Zhou Minghua. \& Wu Xuejun. 2003. Review of Parametric Design Method, Computer Engineering and Applications, (7): 16-18.

[2] Li Bin, Guo Wenxia. \& Zhao Xinhua. 2009. Parameterized design and simulation of parallel manipulator based on ADAMS, Journal of Tianjin University of Technolo$g y, 25(6): 28-30$.

[3] Wang Qi, Cao Fei, Zhang Ren. \& Liu Yukun, 2014. Parameterized Analysis of Palletizing Robot based on ADMAS, Modular Machine Tool \& Automatic Manufacturing Technique, (7): 44-49.

[4] Jiang Libiao. \& Ni Qiang. 2008. Parametric Design and Analysis of Front Independent Suspension of $6 \times 6$ off-road Vehicle with ADMAS/View, Journal of Beijing University of Aeronautics and Astronautics, (2): 249-253.

[5] Jesper S. 2000. Utilization of ADAMS to predict tracked vehicle performance. SAE Technical Paper.

[6] Perter H. \& Philip K. Bertram M. 1998. Suspension kinematics and compliance measuring and simulation. SAE Technical Paper. 980897.

[7] Chen Liping, Mechanical System Dynamics Analysis and ADAMS Application Tutorial. Beijing: Tsinghua University Press.

[8] Meng Xiangxu. \& Xu Yanning, 2002. A Survey of the Research Works on Parametric Design, Journal of Computer-aided Design \& Computer Graphics, 4(11).

[9] Li Zenggang, 2012. Introductory Explanation and Examples by ADAMS, Beijing: National Defend Industry Press, (5)

[10] Gai Yongjun, Pan Chunping. \& Wang Jingquan, 2010. Kinematics Simulation of Parallel Mechanism Based on ADAMS, Journal of Changchun University of Science and Technology (Natural Science Edition), 33 (2): 71-47.

[11]Hu Yong, Ling Mingxiang. \& Wang Jue. 2014. Coupling Dynamical Simulation and Modeling of 2-DOF Parallel Driven Platform, Machine Tool \& Hydraulics, 42 (7). 